PROCEEDINGS OF THE

AMERICAN MATHEMATICAL SOCIETY

Volume 33, Number 2, June 1972

\title{
IMPLICATIVE HOMOMORPHISMS WITH FINITE RANGES
}

\author{
WILLIAM C. NEMITZ
}

\begin{abstract}
In this note, it is shown that every implicative homomorphism with a finite range is a factor of a projection.
\end{abstract}

In this note, we shall prove a theorem to the effect that given any implicative homomorphism with finite range, there is a subimplicative semilattice of the domain on which the homomorphism is an isomorphism. It follows from this that every finite implicative semilattice is an absolute quotient retract, and hence projective. Incidentally, it is easy to show that the only injective implicative semilattice is the trivial (one element) one. The theorem of this note also has applications in the theory of varieties of implicative semilattices, since it follows that the class of all implicative semilattices, which do not contain a given finite subdirectly irreducible implicative semilattice as a subalgebra, is a variety. For definitions and basic properties of implicative semilattices, the reader is referred to [1], [2], [3], and [4].

Let $\langle L, \wedge, *, 1\rangle$ be an implicative semilattice.

DEFINITION 1. If $x$ and $y$ are elements of $L$, we say $y$ is fixed by $x$ if $x * y=y$. If $S \subseteq L$, we say $S$ is fixed by $x$ if every element of $S$ is fixed by $x$. Finally, if $T \subseteq L$, we say $S$ is fixed by $T$ if $S$ is fixed by every element of $T$.

Note that if $S$ is fixed by $T$, then every subset of $S$ is fixed by every subset of $T$. Also, note that if $y$ is fixed by $x$, and $z \in L$, then $z * y$ is fixed by $x$.

LEMMA 1. An element of a bounded implicative semilattice $L$ is closed if and only if it is fixed by every dense element of $L$.

Proof. First let $a$ be closed in $L, d$ dense in $L$. Then $d * a \leqq(d * a)^{* *}=a$, so $a$ is fixed by $d$. Now assume that $a$ is an element of $L$ which is fixed by every dense element of $L$. Then, in particular, $a$ is fixed by $a^{* *} * a$. But $\left(a^{* *} * a\right) * a=\left(a^{* *} * a\right) *\left(a^{* *} \wedge\left(a^{* *} * a\right)\right)=a^{* *}$, since $a^{* *}$ is closed in $L$ and $a^{* * * a}$ is dense. Hence $a$ is closed in $L$.

DEFINITION 2. For $x \in L$, and $S \subseteq L$, we say that $S$ is total with respect to $x$ if $x * y \in S$ for every $y \in S$. Again if $T \subseteq L, S$ is total with respect to $T$ if $S$ is total with respect to every element of $T$.

Received by the editors June 17, 1971 and, in revised form, October 4, 1971.

AMS 1970 subject classifications. Primary 06A25, 06A20.

Key words and phrases. Implicative semilattice, implicative homomorphism, pseudocomplement.

(c. American Mathematical Society 1972 
Note that if $S$ is total with respect to $T$, then $S$ is total with respect to every subset of $T$, and the subimplicative semilattice of $L$ generated by $S$ is total with respect to $T$.

Lemma 2. Let $L$ be an implicative semilattice, $J$ a principal filter of $L$, and $A$ the closed algebra of $J$. Then $A$ is total with respect to $L$, and hence with respect to any subset of $L$.

Proof. This follows from Lemma 1 and the second note after Definition 1.

Definition 3. For $A \subseteq L$, and $B \subseteq L$, let

$$
A \nabla B=\{c: c=a \wedge b \text { for some } a \in A \text { and } b \in B\}
$$

Lemma 3. Let $A \leqq_{s} L, B \leqq_{s} L$, such that $A$ is fixed by $B$, and $B$ is total with respect to $A$. Then $A \nabla B \leqq_{s} L$ ( $\leqq_{s}$ denotes "is a subalgebra of").

Proof. It suffices to show that for $a_{i} \in A, b_{i} \in B, i=1,2,\left(a_{1} \wedge b_{1}\right) *$ $\left(a_{2} \wedge b_{2}\right) \in A \nabla B$. But $\left(a_{1} \wedge b_{1}\right) *\left(a_{2} \wedge b_{2}\right)=b_{1} *\left(a_{1} * a_{2}\right) \wedge a_{1} *\left(b_{1} * b_{2}\right) \in A \nabla B$.

Lemma 4. Let $A \leqq_{s} L, B \leqq_{s} L, C \leqq_{s} L$, such that $A$ is fixed by $B$ and $C$, $B$ is fixed by $C, B$ and $C$ are total with respect to $A$, and $C$ is total with respect to $B$. Then $A$ is fixed by $B \nabla C$, and $B \nabla C$ is total with respect to $A$.

Proof. For $a \in A, b \in B$, and $c \in C,(b \wedge c) * a=b *(c * a)=a$, and $a *(b \wedge c)=a * b \wedge a * c \in B \nabla C$.

Lemma 5. Let $A \leqq_{s} L, B \leqq_{s} L, A^{\prime} \leqq_{s} L^{\prime}, B^{\prime} \leqq_{s} L^{\prime}, L^{\prime}$ another implicative semilattice. Let $A$ be fixed by $B, B$ total with respect to $A$, and similarly for $A^{\prime}$ and $B^{\prime}$. Let $\alpha$ be an isomorphism from $A$ onto $A^{\prime}$, and $\beta$ be an isomorphism from $B$ onto $B^{\prime}$ such that for $a \in A$ and $b \in B$,

$$
\alpha(a) * \beta(b)=\beta(a * b) .
$$

Let $\sigma$ be defined as follows: For $c \in A \Gamma B$, let $c=a \wedge b$, for some $a \in A$ and $b \in B$. Define $\sigma$ by $\sigma(c)=\alpha(a) \wedge \beta(b)$. Then $\sigma$ is an isomorphism from $A \nabla B$ onto $A^{\prime} \nabla B^{\prime}$.

Proof. 1. $\sigma$ is a mapping. Let $c \in A \nabla B$, such that $c=a_{i} \wedge b_{i}, a_{i} \in A$, $b_{i} \in B, i=1,2$. Then $a_{1} \wedge b_{1} \leqq a_{2}$, and so $b_{1} *\left(a_{1} * a_{2}\right)=a_{1} * a_{2}=1$, and hence $a_{1} \leqq a_{2}$. Similarly $a_{2} \leqq a_{1}$, and hence $a_{1}=a_{2}$. Call this element $a$. Now $a * b_{1}=a * b_{2}$, so $\alpha(a) * \beta\left(b_{1}\right)=\beta\left(a * b_{1}\right)=\beta\left(a * b_{2}\right)=x(a) * \beta\left(b_{2}\right)$, and so $\alpha(a) \wedge$ $\beta\left(b_{1}\right)=x(a) \wedge \beta\left(b_{2}\right)$.

2. $\sigma$ is a homomorphism. Clearly, $\sigma$ preserves meets. Let $c_{i}=a_{i} \wedge b_{i}$, 
$a_{i} \in A, b_{i} \in B, i=1,2$. Then

$$
\begin{aligned}
\sigma\left(c_{1} * c_{2}\right) & =\sigma\left(a_{1} * a_{2} \wedge a_{1} *\left(b_{1} * b_{2}\right)\right) \\
& =\alpha\left(a_{1}\right) * \alpha\left(a_{2}\right) \wedge \alpha\left(a_{1}\right) *\left(\beta\left(b_{1}\right) * \beta\left(b_{2}\right)\right) \\
& =\left(\alpha\left(a_{1}\right) \wedge \beta\left(b_{1}\right)\right) *\left(\alpha\left(a_{2}\right) \wedge \beta\left(b_{2}\right)\right) \\
& =\sigma\left(c_{1}\right) * \sigma\left(c_{2}\right) .
\end{aligned}
$$

3. Clearly $\sigma$ is onto.

4. $\sigma$ is one-to-one. Let $\sigma\left(c_{1}\right)=\sigma\left(c_{2}\right), c_{i}=a_{i} \wedge b_{i}, a_{i} \in A, b_{i} \in B, i=1,2$. Then $\alpha\left(a_{1}\right) \wedge \beta\left(b_{1}\right)=\alpha\left(a_{2}\right) \wedge \beta\left(b_{2}\right)$, so $\alpha\left(a_{1}\right)=\alpha\left(a_{2}\right)$, and since $\alpha$ is an isomorphism, $a_{1}=a_{2}$. Also, $\alpha\left(a_{1}\right) * \beta\left(b_{1}\right)=\alpha\left(a_{1}\right) * \beta\left(b_{2}\right)$. So $a_{1} * b_{1}=a_{2} * b_{2}$, and hence $c_{1}=c_{2}$.

THEOREM. Let $f$ be a homomorphism from an implicative semilattice $L$ onto a finite implicative semilattice $M$. Then there is a finite subimplicative semilattice $N$ of $L$ such that $f \mid N$ is an isomorphism onto $M$.

Proof. We first examine the structure of $M$. Since $M$ is bounded, $M=M^{* *} \nabla D_{1}$, where $D_{1}$ is the dense filter of $M$, and $M^{* *}$ is the closed algebra of $M$. Similarly $D_{1}$ can be decomposed into $D_{1}^{* *} \nabla D_{2}$, and so on. Hence, letting $D_{0}=M$,

$$
M=D_{0}^{* *} \nabla\left(D_{1}^{* *} \nabla\left(D_{2}^{* *} \nabla\left(\cdots D_{i}^{* *} \nabla\left(D_{i+1}^{* *} \nabla \cdots \nabla D_{n}\right) \cdots\right)\right)\right) \text {. }
$$

Now each of the $D_{i}^{* *}$ in this sequence is a finite Boolean algebra which is total with respect to all of its predecessors and fixed by each of its successors. That $D_{i}^{* *}$ is total with respect to its predecessors follows from Lemma 2. That $D_{1}^{* *}$ is fixed by each of its successors follows from the fact that $D_{i}^{* *}$ is fixed by $D_{i+1}$ and each of the successors is a subset of $D_{i+1}$.

We shall construct a sequence $E_{0}, E_{1}, E_{2}, \cdots, E_{n}$ such that each $E_{i}$ is a subimplicative semilattice of $L$, and such that

1. $f \mid E_{i}$ is an isomorphism of $E_{i}$ onto $D_{i}^{* *}$,

2. each $E_{i}$ is fixed by each of its successors,

3. each $E_{i}$ is total with respect to each of its predecessors.

The proof is completed by repeatedly applying Lemmas 4 and 5 .

To find the $E_{i}$, first find a principal filter $F_{0}$ of $L$ which $f$ maps onto $D_{0}=$ $M$. Then select a principal filter $F_{1}$ in the dense filter of $F_{0}$ such that $f$ maps $F_{1}$ onto $D_{1}$. Continue in this manner, getting a sequence $F_{0}, F_{1}, \cdots$, $F_{i}, \cdots, F_{n}$, such that $F_{i}$ is a principal filter of the dense filter of $F_{i-1}$, and $f$ maps $F_{i}$ onto $D_{i}$. Each $E_{i}$ will be chosen from the closed algebra of $F_{i}$, and hence clearly condition 2 above will be satisfied.

We shall describe a process for finding $E_{i}$, assuming the $E_{0}, \cdots, E_{i-1}$ have already been found. It will be obvious that a simplification of this process can be used to find $E_{0}$. Briefly, the process is as follows: For each dual atom of $D_{i}^{* *}$, select a preimage under $f$, closed in $F_{i}$. If this preimage fails to meet certain requirements, it is replaced by another preimage 
determined by the first choice. This process of replacement is continued through several steps until a set of preimages of the dual atoms of $D_{i}^{* *}$ is found which generate an algbra $E_{i}$, which satisfies the three requirements stated above.

Let $A=\bigcup_{j=0}^{i-1} E_{j}$. The problem is to find $E_{i}$ total with respect to $A$. For each element $a$ of $A$, let $a^{\prime}$ denote $f(a)$. Now let $b^{\prime}$ be a dual atom of $D_{i}^{* *}$. The elements of $A$ can be divided into two classes; class $1 b^{\prime}$, those elements $a$ of $A$ such that $a^{\prime} * b^{\prime}=1$, and class $2 b^{\prime}$, those elements $a$ of $A$ such that $a^{\prime} * b^{\prime}=b^{\prime}$. These two sets form a partition of $A$, since $a^{\prime} * b^{\prime}$ is an element of $D_{i}^{* *}$. Recall that it follows from Lemma 2 that $D_{i}^{* *}$ is total with respect to $M$. Choose $b \in F_{i}^{* *} \cap f^{-1}\left(b^{\prime}\right)$. Now consider the elements $a$ of class $1 b^{\prime}$ seriatum. If $a * b \neq 1$, replace $b$ by the closure in $F_{i}$ of the pseudojoin (see [5]) of $a$ and $b$. The pseudojoin of $a$ and $b$ is defined to be $((a * b) * b) \wedge$ $((b * a) * a)$. It is idempotent, commutative, an upper bound of the set $\{a, b\}$, and is equal to $b$ if and only if $a \leqq b$. Note that the new $b$ is in $f^{-1}\left(b^{\prime}\right)$. Now let $x$ be the meet of all elements of class $2 b^{\prime}$. If $x * b \neq b$, replace $b$ by $x * b$. At this stage, for each dual atom $b^{\prime}$ of $D_{i}^{* *}$, we have chosen an element $b$ of $F_{i}^{* *} \cap f^{-1}\left(b^{\prime}\right)$, such that if $a$ is in class $1 b^{\prime}$, then $a * b=1$, and if $a$ is in class $2 b^{\prime}, a * b=b$. Hence the set made up of these elements $b$ together with 1 is total with respect to $A$, and thus so is the subimplicative semilattice $B$ generated by this set. Also note that $B$ is finite, $B \subseteq F_{i}^{* *}$, and $f(B)=D_{i}^{* *}$. Now for each dual atom $b^{\prime}$ of $D_{i}^{* *}$ choose $b$ maximal in $B \cap f^{-1}\left(b^{\prime}\right)$. It follows that $b$ is greatest in $B \cap f^{-1}\left(b^{\prime}\right)$, since if $a \in B \cap f^{-1}\left(b^{\prime}\right)$, then so is the pseudojoin of $a$ and $b$. Since $b$ is maximal in $B \cap f^{-1}\left(b^{\prime}\right)$, this pseudojoin equals $b$, and hence $a \leqq b$. Again if $a$ is in class $1 b^{\prime}$, then $a * b=1$, and if $a$ is in class $2 b^{\prime}$, then $a * b=b$. Also, if $b_{1}$ and $b_{2}$ are the elements chosen for two distinct dual atoms $b_{1}^{\prime}$ and $b_{2}^{\prime}$, then $b_{1} * b_{2}=b_{2}$. From this it follows that $E_{i}$ can be taken as the set of elements which can be obtained as meets of some set of these finally chosen $b$, together with 1 .

\section{REFERENCES}

1. Garrett Birkhoff, Lattice theory, 3rd ed., Amer. Math. Soc. Colloq. Publ., vol. 25, Amer. Math. Soc., Providence, R.I., 1967. MR 37 \#2638.

2. H. B. Curry, Foundations of mathematical logic, McGraw-Hill, New York, 1963. MR 26 \#6036.

3. Orrin Frink, Pseudo-complements in semi-lattices, Duke Math. J. 29 (1962), 505514. MR 25 \#3869.

4. W. C. Nemitz, Implicative semi-lattices, Trans. Amer. Math. Soc. 117 (1965), 128142. MR 31 \#1212.

5. - Semi-Boolean lattices, Notre Dame J. Formal Logic 10 (1969), 235-238. MR 39 \#6794.

Department of Mathematics, Southwestern at Memphis, Memphis, Tennessee 38112 